\title{
ER stress in adipocytes and insulin resistance: Mechanisms and significance (Review)
}

\author{
SHAHZAD KHAN and CHANG HUA WANG \\ Department of Pathophysiology, Wuhan University School of Medicine, Wuhan, Hubei 430071, P.R. China
}

Received August 12, 2013; Accepted June 17, 2014

DOI: $10.3892 / \mathrm{mmr} .2014 .2532$

\begin{abstract}
Adipose tissue (AT) has numerous important functions within the body. Of particular importance is its role as an endocrine organ in the control of whole-body glucose and lipid metabolism, which is achieved by the secretion of various proteins. Among these proteins are adipokines, such as adiponectin, leptin, resistin, interleukin- 6 and tumor necrosis factor- $\alpha$. An imbalance in the expression of these adipokines occurs in a variety of conditions, such as obesity, and can lead to various metabolic abnormalities, including hyperglycemia and hyperlipidemia. In turn, this can contribute to insulin resistance (IR) and heart diseases. Adipose endoplasmic reticulum (ER) stress is increasingly recognized as the primary factor governing these conditions, which ultimately result in the initiation of IR or the aggravation of pre-existing IR. Studies have suggested that a number of conditions, including obesity, nutrient overload and metabolic syndromes, can initiate or enhance this process in a multi-dimensional manner. This review focuses on the mechanism by which ER stress in AT can contribute to IR.
\end{abstract}

Correspondence to: Dr Shahzad Khan, Department of Pathophysiology, Wuhan University School of Medicine, 135 Donghu Road, Wuhan, Hubei 430071, P.R. China

E-mail: shahzadkhan571@gmail.com

Abbreviations: ASK1, apoptosis signal-regulating kinase 1; AT, adipose tissue; ATF, activating transcription factor; ADHASC, adult-derived human adipocyte stem cell; CHOP, C/EBP homologous protein; DsbA-L, disulfide-bond A oxidoreductase-like protein; ERK1/2, extracellular signal-regulated kinase 1/2; eIF2 $\alpha$, eukaryotic translation initiation factor; ER, endoplasmic reticulum; ERAD, ER-associated degradation; FFAs, free fatty acids; IKK- $\beta$, IкB kinase- $\beta$; IRE, inositol-requiring enzyme; IRS, insulin receptor substrate; JNK, c-jun N-terminal kinase; MCP, monocyte chemo-attractant protein; MAPK, mitogen-activated protein kinases; $\mathrm{NF \kappa B}$, nuclear factor $\kappa$-light-chain-enhancer of activated B cells; PKR, protein kinase R; PERK, protein kinase R-like ER-regulated kinase; PKA, protein kinase A; ROS, reactive oxygen species; SOCS, suppressor of cytokine signaling; TUDCA, taurine-conjugated ursodeoxycholic acid; TRAF2, tumor necrosis factor receptor-associated factor 2; UPR, unfolded protein response; $\mathrm{XBP} 1, \mathrm{X}$-box binding protein 1

Key words: adipose, insulin resistance, ER stress, adipokines, FFAs

\section{Contents}

1. Endoplasmic reticulum (ER) stress

2. ER stress and the unfolded protein response

3. ER stress in adipose tissue

4. Insulin resistance (IR) in brief

5. IR at the molecular level

6. Adipocyte ER stress and IR: Mechanisms and significance

7. Role of the IRE1-JNK-IRS-1 signaling pathway

8. Role of adipokines

9. Role of inflammatory cytokines during ER stress with IR

10. Role of disulfide-bond A oxidoreductase-like protein

11. A direct role for ER stress in insulin signaling in adipose tissue

12. Role of free fatty acids

13. Role of reactive oxygen species and ER stress in adipocytes

14. Conclusion and future directions

\section{Endoplasmic reticulum (ER) stress}

The ER is a key organelle in cell survival and normal cellular function. In the ER, nascent proteins are folded with the assistance of molecular chaperones and folding enzymes. Correctly folded proteins are subsequently transported to the Golgi apparatus, whereas unfolded and misfolded protein are retained in the ER or returned to the cytoplasm by the process of ER-associated degradation (ERAD) and ultimately degraded by the proteasome. Various factors have been reported to contribute to ER stress, including: i) Disturbances in cellular redox regulation, as a consequence of oxidants, hypoxia or reducing agents, which affect the disulfide bonds of proteins in the ER lumen; ii) glucose deprivation, which disrupts N-linked protein glycosylation in the ER; iii) disruption of $\mathrm{Ca}^{2+}$, which impairs the functions of $\mathrm{Ca}^{2+}$-dependent chaperones, including glucose-regulated protein (GRP) 78 , GRP94 and calreticulin; iv) viral infections which result in the presence of virus-encoded proteins; v) a high-fat diet and vi) protein mutations (1). ER stress activates a stress response signaling network known as the unfolded protein response (UPR), which is capable of inducing apoptosis and inflammatory reactions (2). Of note, the ER also has a role in lipid biosynthesis, including lipid membrane synthesis and controlling the synthesis of cholesterol and other membrane lipid components (3). 


\section{ER stress and the UPR}

The UPR is mediated by at least three transmembrane proteins, including inositol-requiring enzyme 1 (IRE1), protein kinase $\mathrm{R}$ (PKR)-like ER kinase (PERK) and activating transcription factor 6 (ATF6) $(2,4)$. Under unstressed conditions, ER stress transducers, predominantly IRE1, PERK and ATF6, are maintained in an inactive state by binding to the major ER chaperone GRP78 at the ER lumen periphery. Under conditions leading to ER stress, GRP78 is displaced to interact with misfolded luminal proteins, resulting in the release and activation of IRE1, PERK and ATF6. In ER stress, PERK phosphorylates the $\alpha$ subunit of eukaryotic translation initiation factor 2 (eIF2 $\alpha$ ), leading to a rapid reduction in the initiation of mRNA translation and a reduction in the level of newly synthesized proteins in the ER. However, eIF2 $\alpha$ phosphorylation by PERK enables the translation of ATF4. ATF4 promotes apoptosis by inducing the transcription of genes involved in apoptosis, including tribbles homolog 3 and $\mathrm{C} / \mathrm{EBP}$ homologous protein (CHOP).

ER stress-induced IRE1 activation initiates non-spliceosomal splicing of the mRNA of the transcription factor X-box binding protein 1 (XBP1). XBP1 controls protective responses to ER stress, for example by upregulating the transcription of genes encoding ER chaperones and ERAD components. Using its non-specific endoribonuclease activity, IRE1 induces the degradation of ER-localized mRNAs, a process known as regulated IRE1-dependent decay (5). This process further reduces protein accumulation in the ER. Of note, IRE1 also activates c-Jun N-terminal kinase (JNK) by recruiting the scaffold protein tumor necrosis factor receptor-associated factor 2 (TRAF2), as well as apoptosis signal-regulating kinase (ASK1) and caspase $12(2,6,7)$.

Following translocation to the Golgi apparatus under ER stress, ATF6 is cleaved by site 1 and site 2 proteases in the process of regulated intramembrane proteolysis. The cytoplasmic region of ATF6 is an active transcription factor responsible for the transactivation of various genes encoding ER chaperones, ER AD components and protein foldases. ATF6 and PERK also activate nuclear factor $\kappa$-light-chain-enhancer of activated B cells $(\mathrm{NF} \kappa \mathrm{B})$ during ER stress (8-11). Increased levels of JNK and NFKB promote the production of pro-inflammatory cytokines, which cause further inflammation and ER stress (12).

\section{ER stress in adipose tissue (AT)}

Adipose tissue is increasingly recognized as a tissue containing a molecular network that connects obesity, adipokine secretion, chronic inflammation and insulin resistance (IR). In AT, adipocytes have been found to constitute $\leq 50 \%$ of the total number of cells, with other cell types including preadipocytes, macrophages and vascular cells. Under conditions of ER stress, AT secretes a number of protein signals and factors. A previous study showed that UPR markers are overexpressed in the AT of obese rodents (13). In adult-derived human adipocyte stem cells (ADHASCs), ER stress has been reported to increase the levels of ER stress genes and eIF $2 \alpha$ phosphorylation (14). Furthermore, AT inflammation, which is observed in obesity and diabetes, is associated with the infiltration of macrophages into the AT.
This may be triggered by adipocyte death, the secretion of adipokines, including tumor necrosis factor- $\alpha$ (TNF- $\alpha$ ) and interleukin-6 (IL-6), and increased levels of adipocyte-associated chemokines, including monocyte chemoattractant protein 1 (MCP-1). Similar to other cells that demonstrate a high secretory capacity, including mature B lymphocytes, liver cells and pancreatic $\beta$ cells, adipocytes have also been reported to adapt their ER capabilities under conditions of stress, particularly in diabetes and obesity (15). ER stress has been observed in human AT in numerous studies, indicating that ER stress may play a crucial role in AT disorders, including inflammation and apoptosis (16-18). In obesity, the AT is poorly oxygenated $(19,20)$, which leads to AT hypoxia. This interferes with disulfide bonding in the ER lumen, resulting in ER stress, initiation of the UPR and increased levels of ER stress markers in the AT, including CHOP and GRP78 (13,21-23). Hosogai et al (22) reported that, in 3T3-L1 adipocytes, hypoxia is associated with ER stress and increased levels of GRP78 and CHOP. Furthermore, using ATF4, GADD34 and ATF3 as markers of the apoptotic pathway, Sharma et al (17) found that ER stress induced apoptosis. Hypoxia does not only stimulate the inflammatory response of macrophages $(24,25)$, but also induces apoptosis and $\mathrm{G}_{0} / \mathrm{G}_{1}$-phase cell cycle arrest through AKT and JNK (26). Furthermore, Yin et al (27) found that hypoxia induces cell death by promoting the release of free fatty acids (FFAs) and inhibiting glucose uptake in adipocytes via the inhibition of the insulin signaling pathway.

\section{IR in brief}

Prior to discussing the association between IR and AT ER stress, an understanding of IR is required. IR is caused by an impaired sensitivity of target organs, including AT, the liver and muscle, to insulin. Insulin regulates glucose uptake in the liver and muscle. Furthermore, insulin is a key regulator of circulating FFA concentrations. In AT, insulin decreases lipolysis and thereby reduces FFA efflux from adipocytes; in skeletal muscle, insulin predominantly induces glucose uptake by stimulating the translocation of glucose transporter type 4 (GLUT4) to the plasma membrane. Furthermore, in the liver, insulin inhibits gluconeogenesis by reducing the activity of key enzymes. Therefore IR has been suggested to increase circulating FFA concentrations, leading to ectopic fat deposition that impedes insulin-mediated glucose uptake in skeletal muscle and increases glucose generation in the liver (28). A combination of IR and abnormalities in insulin secretion can lead to Type 2 diabetes mellitus (T2DM).

\section{IR at the molecular level}

In normal conditions, insulin is associated with complex signaling cascades. Briefly, insulin receptor-mediated tyrosine phosphorylation of insulin receptor substrates (IRSs) induces the activation of at least two major pathways: The phosphatidylinositol 3-kinase (PI3K)-AKT and mitogen-activated protein kinase (MAPK) pathways. The PI3K-AKT pathway is primarily responsible for the effect of insulin on glucose uptake and the suppression of gluconeogenesis, whereas the MAPK pathway regulates gene expression and interacts with 
the PI3K-AKT pathway to control cell growth and differentiation.

While tyrosine phosphorylation activates, serine phosphorylation of IRSs at specific serine residues inhibits insulin signaling. IкB kinase- $\beta$ (IKK- $\beta$ ), JNK 1 and MAPKs are examples of serine kinases that phosphorylate IRS1 and consequently inactivate its insulin signaling activity. Of note, these serine kinases are also mediators of inflammatory signaling pathways, demonstrating that an inhibitory crosstalk may exist between inflammatory and insulin signaling at a molecular level. The association between cytokine signaling and the inhibition of insulin signaling has also been indicated by the presence of molecular mediators, including suppressor of cytokine signaling (SOCS) 1 and 3 and nitric oxide (NO). IL-6 has been shown to induce the activation of SOCS proteins during inflammation, causing the ubiquitinylation and degradation of IRS proteins. Endogenous NO production by inducible NO synthase (iNOS), under the action of numerous inflammatory cytokines, can limit IRS1 and iNOS activity, resulting in reduced AKT activity; AKT is a key mediator of IRS signaling (29).

\section{Adipocyte ER stress and IR: Mechanisms and significance}

ER stress was first observed by Ozcan et al (13) in the AT of obese mice and proposed as a risk factor for IR. It has since been demonstrated that stress-inducing conditions, such as obesity, are not only associated with ER stress and the apoptosis of $\beta$-cells, hepatocytes and adipocytes, but also with metabolic disorders, particularly IR. In patients with IR, treatment with taurine-conjugated ursodeoxycholic acid (TUDCA), a conjugated bile acid derivative that inhibits ER stress-induced apoptosis, was observed to increase insulin sensitivity (30). Furthermore, the administration of chaperones, including 4-phenyl butyric acid (PBA), trimethylamine $\mathrm{N}$-oxide dihydrate, dimethyl sulfoxide and $150 \mathrm{kDa}$ oxygen-regulated protein, which protect cells from ER stress by stabilizing protein conformation and improving ER folding capacity, was found to increase insulin sensitivity in obese diabetic mice $(31,32)$. These data indicate that ER stress in adipocytes, hepatocytes and $\beta$-cells may, at least in part, initiate IR, as well as aggravate pre-existing IR, particularly in the context of obesity. The mechanism by which ER stress interferes with insulin receptor signaling is multifactorial, and the role of adipose cells/AT in IR is discussed later in this review.

\section{Role of the IRE1-JNK-IRS-1 signaling pathway}

It has been suggested that ER stress in adipocytes may disrupt insulin signaling through the activation of IRE1. ER stress induces insulin receptor signaling through increasing the serine phosphorylation and decreasing the tyrosine phosphorylation of IRS-1, leading to IR (33). As mentioned previously, IRS-1 becomes inactivated upon phosphorylation of specific serine residues (34). Several intracellular serine kinases may mediate this IRS-1 phosphorylation, including IKK, JNK, mammalian target of rapamycin (mTOR) and protein kinase $\mathrm{C}-\theta(\mathrm{PKC}-\theta)$. Insulin-resistant states, including obesity and T2DM, are associated with the activation of
JNK and/or IKK, which leads to serine phosphorylation of IRS1 and thus the induction of IR (35-39). In obesity, JNK is activated by IRE1 kinase activity as a consequence of AT ER. Furthermore, PKR, which is activated by saturated FAs, ceramides and lipopolysaccharide, is capable of inhibiting insulin signaling directly by phosphorylating IRS1 on serine residues or indirectly by stimulating JNK activity (40). The IRE1-JNK signaling pathway has also been reported to directly inhibit cytoplasmic insulin signaling in ob/ob mice due to JNK-mediated phosphorylation of IRS-1 at serine-307 (13). Additionally, the inflammatory cytokines secreted by adipocytes and macrophages in AT in obese patients can activate JNK pathways (41). Furthermore, inflammatory cytokines can impair insulin signaling by interfering with IRS-1-insulin receptor interactions and promoting IRS-1 degradation (42). However, the effect of ER stress on insulin signaling requires further investigation in order to elucidate the specific mechanisms underlying the development of IR in AT in obese patients. In a study on AT from obese volunteers, approximately a two-fold upregulation of phosphorylated JNK-1 and an upregulation of the spliced form of XBP1, which is part of the IRE-1/XBP1 proximal ER stress sensor, was observed in the AT (43) (Fig. 1).

\section{Role of adipokines}

Several investigations in rodents and humans have found an association between IR and ER stress in AT, as well as increased lipolysis and altered adipokine production $(13,16-18,44)$. In ER stress-associated conditions, including obesity, a decrease in leptin and adiponectin secretion and an increase in IL-6 secretion may be observed (44). Furthermore, studies have shown that adiponectin folding and multimerization are impaired in obese states due to a decreased expression of ER disulfide-bond A oxidoreductase-like protein (DsbA-L), which leads to ER stress $(45,46)$. However, CHOP is upregulated under ER stress, which impairs resistin transcription in adipocytes and alters its secretion (47). Of note, a number of studies have shown that a decreased expression of the cytokine adiponectin may promote IR in obesity (48-50). ER stress has been reported to decrease levels of high-molecular-weight fractions as well as total adiponectin in human adipocytes, which may have role in insulin sensitivity and metabolic syndrome $(51,52)$. Decreased levels of adiponectin are observed in conditions associated with excessive nutrient intake, including obesity. Adiponectin signaling targets adenosine monophosphate-activated protein kinase, which is a negative regulator of mTOR. High levels of mTOR in turn induce serine phosphorylation of IRS1. Therefore, when adiponectin levels are low, the inhibition of IRS1 causes IR through the activation of the mTOR signaling pathway. An investigation using tunicamycin and thapsigargin as ER stress inducers in ADHASCs found that ER stress decreased adiponectin and increased TNF- $\alpha$ mRNA expression, in addition to decreasing levels of inhibitor of $\mathrm{NF \kappa B}-\alpha$ (IкB) protein (14).

Resistin is an adipokine secreted by preadipocytes of human AT (53) and has been associated with IR. ER stress has been found to reduce resistin mRNA expression in 3T3-L1 adipocytes in a time- and dose-dependent manner, indicating that resistin is regulated by ER stress (47). Furthermore, in 


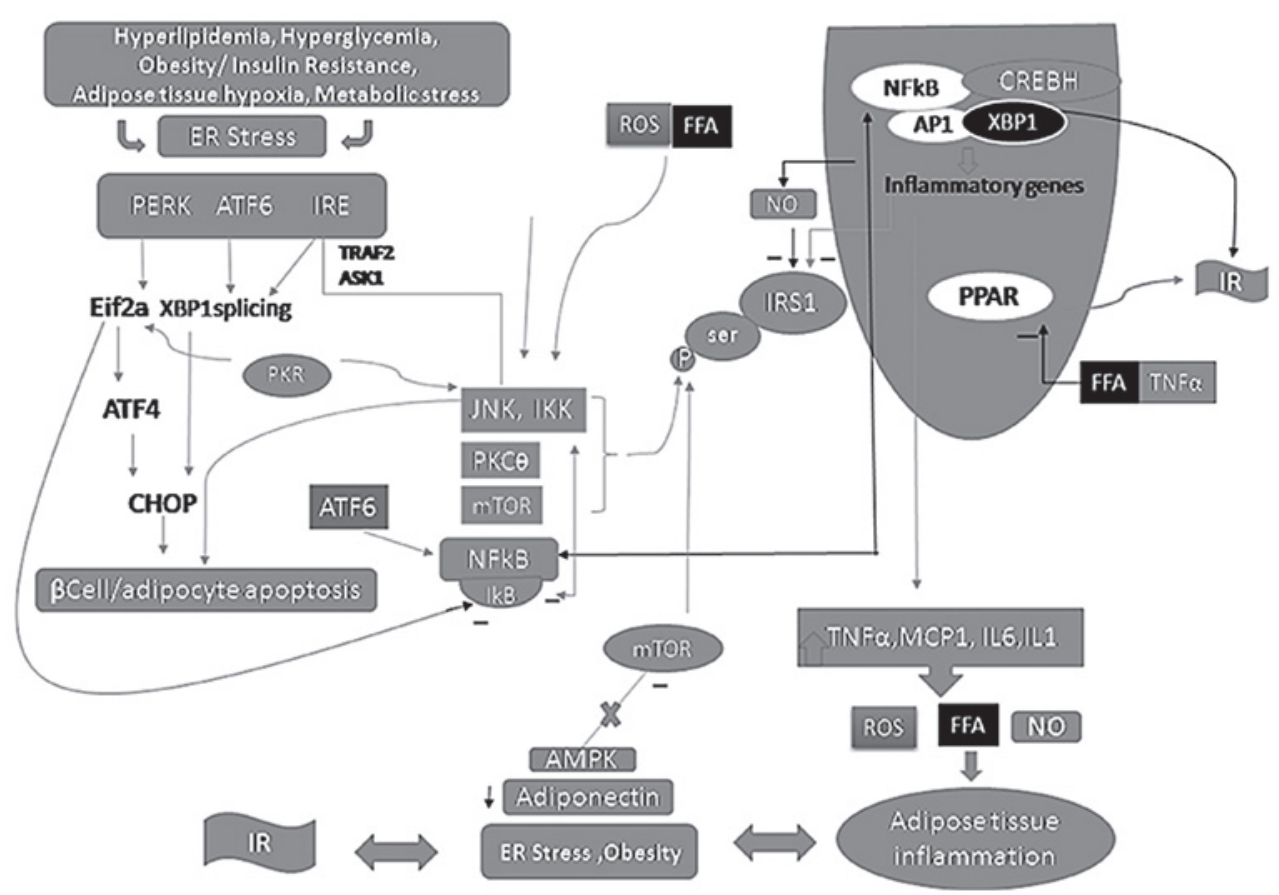

Figure 1. Association between ER stress in adipocytes and IR. The process is multifactorial and highly interlinked. ER, endoplasmic reticulum; IR, insulin resistance; ROS, reactive oxygen species; FFA, free fatty acid; NO, nitric oxide.

adipocytes, ER stress affects IR through resistin. However, further investigations are required as resistin was observed to be positively correlated with IR in a study involving individuals who were obese and diabetic (54), while no correlation was observed in other studies involving individuals with a normal weight and children who were obese (55-57).

\section{Role of inflammatory cytokines during ER stress with IR}

In a study by Hotamisligil (58), inflammation was observed to induce IR through the inhibition of IRS in the insulin signaling pathway. Furthermore, inflammation and ER stress in AT are known to exhibit a dual relationship. Inflammation can cause ER stress in AT, while ER stress, either due to obesity or obesity-associated consequences, such as IR and increased FFA levels, can increase the activity of anti- or pro-inflammatory proteins. Studies have shown that obese patients exhibit increased plasma levels of C-reactive protein, inflammatory cytokines, including TNF- $\alpha$, IL-6, MCP-1 and IL-8, and the multifunctional proteins leptin (59) and osteopontin $(60,61)$, indicating a continuous low-grade inflammation in the obese state.

All three subdivisions of ER stress associated with the UPR contribute to the low-grade inflammation associated with obesity. Of note, IRE1 activates JNK and NFKB through TRAF2 and ASK1. In addition, PERK inhibits IאB through eIF2 $\alpha$ phosphorylation. PKR also phosphorylates eIF2 $\alpha$ and activates JNK and IKK, while the ATF6 branch of the pathway activates NFkB. Finally, ER stress activates cyclic adenosine monophosphate (cAMP) responsive element-binding protein, hepatocyte-specific, which, together with $\mathrm{NF \kappa B}$, augments the transcription of genes involved in inflammation $(38,62)$. The role of IKB and IKK in ER-induced inflammation has been investigated in ADHASCs. The IкB family of proteins controls the activation of $\mathrm{NF \kappa B}$, whose entry into the nucleus can initiate inflammation. Therefore, IкB regulates $\mathrm{NF \kappa B}$ by sequestering it in the cytoplasm. In turn, IкB can be degraded by IKK, which results in entry of the NFKB dimers into the nucleus and inflammation. Therefore, ER stress may induce inflammation through a decrease in I $\kappa \mathrm{B}$ levels as a consequence of increased IKK activity (58).

IL- 6 and TNF- $\alpha$, levels of which are increased in obesity and IR, have been proposed to induce ER stress and consequently promote IR in a positive feedback manner (63). In ATs, TNF- $\alpha$ inhibits lipogenesis and adiponectin expression via the inhibition of peroxisome proliferator-activated receptor- $\gamma$ (PPAR- $\gamma$ )-mediated mechanisms (64-66). It has been suggested that ER stress may activate TNF- $\alpha$, which is then involved in inflammatory processes and the PPAR- $\gamma$-mediated effects on adipocytes (67). Investigations using PPAR activators such as thiazolidinediones have verified that the transcriptional activity of PPAR- $\gamma$ is required for the maintenance of insulin sensitivity and lipid metabolism (26). A previous study investigated TNF- $\alpha$ in the AT of lean and obese subjects using reverse transcription-polymerase chain reaction methodology (43). Neutralizing TNF- $\alpha$ in rats and knocking out the TNF- $\alpha$ or TNF- $\alpha$ receptor 1 genes in mice have also shown protective effects against IR induced by diet and genetic obesity $(68,69)$. Furthermore, TNF- $\alpha$ activates several IR-related pathways, including IKK- $\beta$ and SOCS3, in cultured murine adipocytes $(70,71)$. TNF- $\alpha$ has been suggested to affect insulin sensitivity by modifying the expression of IRS1, GLUT4, adiponectin and PPAR- $\alpha(66,72)$. Of note, TNF- $\alpha$ also induces reactive oxygen species (ROS) generation through the activation of the nicotinamide adenine dinucleotide phosphate-oxidase (NADPH oxidase) $(73,74)$. In addition, another inflammatory cytokine, IL-1 $\beta$, plays an important role in inducing ER stress in adipocytes in obese individuals by increasing the levels of iNOS, which generates 
NO. In turn, NO inhibits the activity of the ER $\mathrm{Ca}^{2+}$ pump, resulting in a depletion of $\mathrm{ER} \mathrm{Ca}^{2+}$ stores, and thus inducing ER stress (75,76).

Obesity-induced ER stress was recently investigated in AT using the 12/15-lipoxygenase (12/15-LO) enzyme, which is involved in a unique inflammatory pathway that regulates the ER stress response in key cells, tissues and organs, including adipocytes, pancreatic islets and the liver (77). The addition of the 12/15-LO cleavage products, 12-hydroxyeicosatetraenoic acid and 12-hydroperoxyeicosatetraenoic acid, to differentiated 3T3-L1 adipocytes was observed to induce the expression and activation of ER stress markers, including GRP78, XBP1, phosphorylated-PERK and phosphorylated-IRE1 $\alpha$. The study also found that 12/15-LO upregulated IL-12 expression. These findings may represent a novel therapeutic strategy for alleviating ER-stress associated inflammation, $\beta$-cell dysfunction and IR, thereby reducing metabolic complications associated with visceral adiposity by inhibiting $12 / 15$-LO activation or downstream IL-12 signaling (77).

\section{Role of DsbA-L}

DsbA-L is a protein that has been proposed to have a role in the downregulation of adiponectin during ER stress-induced autophagy in adipocytes (78). It was revealed that ER stress-induced autophagy plays an important role in obesity-induced adiponectin downregulation in adipocytes, and that increasing the expression of DsbA-L may increase adiponectin levels and lead to enhanced insulin sensitivity in vitro and in vivo. Therefore, increasing DsbA-L expression levels could be a novel approach to protect cells from obesity-induced ER stress and improve insulin sensitivity. Of note, cellular DsbA-L levels were stimulated by the PPAR- $\gamma$ agonist rosiglitazone, an insulin-sensitizing drug (78).

\section{A direct role for ER stress in insulin signaling in AT}

$\mathrm{Xu}$ et al (44) demonstrated a direct role for ER stress in insulin signaling in adipose cells. ER stress inducers were observed to decrease insulin signaling in 3T3-L1 adipocytes without affecting insulin-stimulated glucose uptake (79), in a manner independent of the IRE1/JNK pathway (44). Notably, ER stress has also been revealed to directly increase lipolysis by downregulating the expression of the lipid droplet-associated protein perilipin A $(80,81)$.

\section{Role of FFAs}

The roles of hypoxia and inflammatory mediators in AT ER stress have been discussed previously in this review. A number of studies have demonstrated that elevated FFA levels may also have an important role in the induction of ER stress in various cells, including adipocytes $(62,82)$; however, further investigation is required. This theory is supported by the fact that numerous obese individuals exhibit elevated FFA plasma levels $(83,84)$. Hotamisligil (58) showed that inflammation may lead to IR by inhibiting IRSs in the insulin signaling pathway, and it is well established that inflammation inhibits the action of insulin by increasing the levels of FFAs and decreasing those of adiponectin in the blood. Therefore, it is possible that both inflammatory cytokines and FFAs may target IRSs, leading to IR $(85,86)$. High FFA levels have also been shown to downregulate PPAR- $\gamma$ protein and mRNA expression, further enhancing IR (87). In AT, ER stress promotes FFA efflux from adipocytes, and high levels of circulating FFA have been suggested to be the cellular basis of lipotoxicity, dyslipidemia and IR $(13,17,88-90)$. Furthermore, accumulating evidence suggests that saturated long-chain FFAs, primarily palmitate, and, to a lesser extent, unsaturated long chain FFAs may induce ER stress and mediate $\beta$-cell apoptosis, ultimately leading to IR and T2DM (91-96). In a recent study, a unique lipolysis pathway was reported that occurred in response to ER stress in adipocytes. This pathway occurred independently of hormone-sensitive lipolysis, but was associated with elevated cAMP production and PKA activity (81). Chemically induced ER stress was revealed to activate cAMP/PKA and extracellular signal-regulated kinase 1/2 (ERK1/2) and regulate lipolysis in ER-stressed adipocytes, with PKA being an acute regulator and ERK1/2 a chronic regulator (81). Notably, it has been suggested that JNK (97) and PKC (98) may also modulate lipolysis and that ERK1/2 and JNK are activated during ER stress.

\section{Role of ROS and ER stress in adipocytes}

ROS have been reported to play an important role in the ER stress response in adipocytes, which then directly or indirectly contributes to IR (21). Increased ROS generation has also been observed in response to high levels of FFAs in the AT of obese mice. Furthermore, TNF- $\alpha$ has been shown to induce ROS generation through the activation of NADPH oxidase $(74,75,99)$. As a consequence of their oxidizing effects on nascent proteins and their action on $\mathrm{Ca}^{2+}$ channels, ROS lower $\mathrm{Ca}^{2+}$ availability and increase the number of misfolded and unfolded proteins in the ER, which further increases ER stress $(39,100)$. This FFA-mediated ROS generation model provides another mechanism by which ER stress may be induced in AT and subsequently lead to IR.

\section{Conclusion and future directions}

In conclusion, this review demonstrates the existence of a strong association between adipocyte ER stress and IR that is complex and multifactorial. Therefore, the inhibition of ER stress may lead to the discovery of novel therapeutics for the treatment of metabolic diseases, including T2DM; investigations are currently underway in this area. To date, at least two chaperones, PBA and TUDCA, have been approved by the Food and Drug Administration and have been shown to relieve ER stress-mediated pathologies, including IR, in hepatocytes, adipocytes and $\beta$-cells $(31,32,94)$. However, further investigations are required before these strategies can be applied in patients for the treatment of metabolic and nutritional disorders, including obesity and IR.

\section{References}

1. Schröder M: Endoplasmic reticulum stress responses. Cell Mol Life Sci 65: 862-894, 2008.

2. Winnay JN and Kahn CR: PI 3-kinase regulatory subunits as regulators of the unfolded protein response. Methods Enzymol 490: 147-158, 2011. 
3. van der Kallen CJ, van Greevenbroek MM, Stehouwer CD and Schalkwijk CG: Endoplasmic reticulum stress-induced apoptosis in the development of diabetes: is there a role for adipose tissue and liver? Apoptosis 14: 1424-1434, 2009

4. Schröder M and Kaufman RJ: The mammalian unfolded protein response. Annu Rev Biochem 74: 739-789, 2005.

5. Hollien J and Weissman JS: Decay of endoplasmic reticulum-localized mRNAs during the unfolded protein response. Science 313: 104-107, 2006

6. Hu P, Han Z, Couvillon AD, Kaufman RJ and Exton JH: Autocrine tumor necrosis factor alpha links endoplasmic reticulum stress to the membrane death receptor pathway through IRE1alpha-mediated NF-kappaB activation and down-regulation of TRAF2 expression. Mol Cell Biol 26: 3071-3084, 2006.

7. Urano F, Wang X, Bertolotti A, et al: Coupling of stress in the ER to activation of JNK protein kinases by transmembrane protein kinase IRE1. Science 287: 664-666, 2000.

8. Deng J, Lu PD, Zhang Y, et al: Translational repression mediates activation of nuclear factor kappa B by phosphorylated translation initiation factor 2. Mol Cell Biol 24: 10161-10168, 2004.

9. Jiang HY, Wek SA, McGrath BC, et al: Phosphorylation of the alpha subunit of eukaryotic initiation factor 2 is required for activation of NF-kappaB in response to diverse cellular stresses. Mol Cell Biol 23: 5651-5663, 2003.

10. Wu S, Tan M, Hu Y, Wang JL, Scheuner D and Kaufman RJ: Ultraviolet light activates NFkappaB through translational inhibition of IkappaBalpha synthesis. J Biol Chem 279: 34898-34902, 2004.

11. Yamazaki H, Hiramatsu N, Hayakawa K, et al: Activation of the Akt-NF-kappaB pathway by subtilase cytotoxin through the ATF6 branch of the unfolded protein response. J Immunol 183: 1480-1487, 2009

12. Zhang K, Shen X, Wu J, et al: Endoplasmic reticulum stress activates cleavage of CREBH to induce a systemic inflammatory response. Cell 124: 587-599, 2006

13. Ozcan U, Cao Q, Yilmaz E, et al: Endoplasmic reticulum stress links obesity, insulin action, and type 2 diabetes. Science 306 457-461, 2004

14. Mondal AK, Das SK, Varma V, et al: Effect of endoplasmic reticulum stress on inflammation and adiponectin regulation in human adipocytes. Metab Syndr Relat Disord 10: 297-306, 2012

15. Wu J and Kaufman RJ: From acute ER stress to physiological roles of the Unfolded Protein Response. Cell Death Differ 13 374-384, 2006

16. Boden G, Duan X, Homko C, et al: Increase in endoplasmic reticulum stress-related proteins and genes in adipose tissue of obese, insulin-resistant individuals. Diabetes 57: 2438-2444, 2008

17. Sharma NK, Das SK, Mondal AK, et al: Endoplasmic reticulum stress markers are associated with obesity in nondiabetic subjects. J Clin Endocrinol Metab 93: 4532-4541, 2008

18. Gregor MF, Yang L, Fabbrini E, et al: Endoplasmic reticulum stress is reduced in tissues of obese subjects after weight loss. Diabetes 58: 693-700, 2009.

19. Fleischmann E, Kurz A, Niedermayr M, et al: Tissue oxygenation in obese and non-obese patients during laparoscopy. Obes Surg 15: 813-819, 2005.

20. Virtanen KA, Lönnroth P, Parkkola R, et al: Glucose uptake and perfusion in subcutaneous and visceral adipose tissue during insulin stimulation in nonobese and obese humans. J Clin Endocrinol Metab 87: 3902-3910, 2002

21. Furukawa S, Fujita T, Shimabukuro M, et al: Increased oxidative stress in obesity and its impact on metabolic syndrome. J Clin Invest 114: 1752-1761, 2004.

22. Hosogai N, Fukuhara A, Oshima K, et al: Adipose tissue hypoxia in obesity and its impact on adipocytokine dysregulation. Diabetes 56: 901-911, 2007.

23. Lazar MA: How obesity causes diabetes: not a tall tale Science 307: 373-375, 2005 .

24. Heilbronn LK and Campbell LV: Adipose tissue macrophages, low grade inflammation and insulin resistance in human obesity. Curr Pharm Des 14: 1225-1230, 2008.

25. Lewis JS, Lee JA, Underwood JC, Harris AL and Lewis CE: Macrophage responses to hypoxia: relevance to disease mechanisms. J Leukoc Biol 66: 889-900, 1999.

26. Spiegelman BM: PPAR-gamma: adipogenic regulator and thiazolidinedione receptor. Diabetes 47: 507-514, 1998.

27. Yin J, Gao Z, He Q, Zhou D, Guo Z and Ye J: Role of hypoxia in obesity-induced disorders of glucose and lipid metabolism in adipose tissue. Am J Physiol Endocrinol Metab 296: E333-E342, 2009.
28. Samuel VT and Shulman GI: Mechanisms for insulin resistance: common threads and missing links. Cell 148: 852-871, 2012.

29. Zeyda M and Stulnig TM: Obesity, inflammation, and insulin resistance - a mini-review. Gerontology 55: 379-386, 2009.

30. Kars M, Yang L, Gregor MF, et al: Tauroursodeoxycholic Acid may improve liver and muscle but not adipose tissue insulin sensitivity in obese men and women. Diabetes 59: 1899-1905, 2010.

31. Nakatani Y, Kaneto H, Kawamori D, et al: Involvement of endoplasmic reticulum stress in insulin resistance and diabetes. J Biol Chem 280: 847-851, 2005.

32. Ozcan U, Yilmaz E, Ozcan L, et al: Chemical chaperones reduce ER stress and restore glucose homeostasis in a mouse model of type 2 diabetes. Science 313: 1137-1140, 2006.

33. Kim DS, Jeong SK, Kim HR, Kim DS, Chae SW and Chae HJ: Effects of triglyceride on ER stress and insulin resistance. Biochem Biophys Res Commun 363: 140-145, 2007.

34. Gual P, Le Marchand-Brustel Y and Tanti JF: Positive and negative regulation of insulin signaling through IRS-1 phosphorylation. Biochimie 87: 99-109, 2005.

35. Yuan M, Konstantopoulos N, Lee J, et al: Reversal of obesityand diet-induced insulin resistance with salicylates or targeted disruption of Ikkbeta. Science 293: 1673-1677, 2001.

36. Itani SI, Ruderman NB, Schmieder F and Boden G: Lipid-induced insulin resistance in human muscle is associated with changes in diacylglycerol, protein kinase C, and IkappaB-alpha. Diabetes 51: 2005-2011, 2002.

37. Arkan MC, Hevener AL, Greten FR, et al: IKK-beta links inflammation to obesity-induced insulin resistance. Nat Med 11: 191-198, 2005.

38. Hirosumi J, Tuncman G, Chang L, et al: A central role for JNK in obesity and insulin resistance. Nature 420: 333-336, 2002.

39. Zhang K and Kaufman RJ: From endoplasmic-reticulum stress to the inflammatory response. Nature 454: 455-462, 2008.

40. Nakamura T, Furuhashi M, Li P, et al: Double-stranded RNA-dependent protein kinase links pathogen sensing with stress and metabolic homeostasis. Cell 140: 338-348, 2010.

41. Kyriakis JM and Avruch J: Mammalian mitogen-activated protein kinase signal transduction pathways activated by stress and inflammation. Physiol Rev 81: 807-869, 2001.

42. Shoelson SE, Lee $\mathbf{J}$ and Goldfine AB: Inflammation and insulin resistance. J Clin Invest 116: 1793-1801, 2006.

43. Boden G and Merali S: Measurement of the increase in endoplasmic reticulum stress-related proteins and genes in adipose tissue of obese, insulin-resistant individuals. Methods Enzymol 489: 67-82, 2011.

44. Xu L, Spinas GA and Niessen M: ER stress in adipocytes inhibits insulin signaling, represses lipolysis, and alters the secretion of adipokines without inhibiting glucose transport. Horm Metab Res 42: 643-651, 2010

45. Liu M,Zhou L, Xu A, etal: A disulfide-bond A oxidoreductase-like protein (DsbA-L) regulates adiponectin multimerization. Proc Natl Acad Sci USA 105: 18302-18307, 2008.

46. Zhou L, Liu M, Zhang J, Chen H, Dong LQ and Liu F: DsbA-L alleviates endoplasmic reticulum stress-induced adiponectin downregulation. Diabetes 59: 2809-2816, 2010.

47. Lefterova MI, Mullican SE, Tomaru T, Qatanani M, Schupp M and Lazar MA: Endoplasmic reticulum stress regulates adipocyte resistin expression. Diabetes 58: 1879-1886, 2009.

48. Hu E, Liang P and Spiegelman BM: AdipoQ is a novel adipose-specific gene dysregulated in obesity. J Biol Chem 271: 10697-10703, 1996.

49. Berg AH, Combs TP and Scherer PE: ACRP30/adiponectin: an adipokine regulating glucose and lipid metabolism. Trends Endocrinol Metab 13: 84-89, 2002

50. Menzaghi C, Ercolino T, Di Paola R, et al: A haplotype at the adiponectin locus is associated with obesity and other features of the insulin resistance syndrome. Diabetes 51: 2306-2312, 2002.

51. Kern PA, Di Gregorio GB, Lu T, Rassouli N and Ranganathan G: Adiponectin expression from human adipose tissue: relation to obesity, insulin resistance, and tumor necrosis factor-alpha expression. Diabetes 52: 1779-1785, 2003

52. Lara-Castro C, Luo N, Wallace P, Klein RL and Garvey WT: Adiponectin multimeric complexes and the metabolic syndrome trait cluster. Diabetes 55: 249-259, 2006.

53. Kaser S, Kaser A, Sandhofer A, Ebenbichler CF, Tilg H and Patsch JR: Resistin messenger-RNA expression is increased by proinflammatory cytokines in vitro. Biochem Biophys Res Commun 309: 286-290, 2003. 
54. Silha JV, Krsek M, Skrha JV, Sucharda P, Nyomba BL and Murphy LJ: Plasma resistin, adiponectin and leptin levels in lean and obese subjects: correlations with insulin resistance. Eur J Endocrinol 149: 331-335, 2003

55. Vozarova de Courten B, Degawa-Yamauchi M, Considine RV and Tataranni PA: High serum resistin is associated with an increase in adiposity but not a worsening of insulin resistance in Pima Indians. Diabetes 53: 1279-1284, 2004

56. Lee JH, Chan JL, Yiannakouris N, et al: Circulating resistin levels are not associated with obesity or insulin resistance in humans and are not regulated by fasting or leptin administration: cross-sectional and interventional studies in normal, insulin-resistant, and diabetic subjects. J Clin Endocrinol Metab 88: 4848-4856, 2003.

57. Gherlan I, Vladoiu S, Alexiu F, et al: Adipocytokine profile and insulin resistance in childhood obesity. Maedica (Buchar) 7: 205-213, 2012.

58. Hotamisligil GS: Inflammation and metabolic disorders Nature 444: 860-867, 2006

59. Friedman JM and Halaas JL: Leptin and the regulation of body weight in mammals. Nature 395: 763-770, 1998.

60. Kiefer FW, Zeyda M, Todoric J, et al: Osteopontin expression in human and murine obesity: extensive local up-regulation in adipose tissue but minimal systemic alterations. Endocrinology 149: 1350-1357, 2008.

61. Kahn BB and Flier JS: Obesity and insulin resistance. J Clin Invest 106: 473-481, 2000.

62. Jiao P, Ma J, Feng B, et al: FFA-induced adipocyte inflammation and insulin resistance: involvement of ER stress and IKK $\beta$ pathways. Obesity (Silver Spring) 19: 483-491, 2011

63. Bruun JM, Verdich C, Toubro S, Astrup A and Richelsen B: Association between measures of insulin sensitivity and circulating levels of interleukin-8, interleukin-6 and tumor necrosis factor-alpha. Effect of weight loss in obese men. Eur J Endocrinol 148: 535-542, 2003.

64. Sung HY, Hong CG, Suh YS, et al: Role of (-)-epigallocatechin-3-gallate in cell viability, lipogenesis, and retinol-binding protein 4 expression in adipocytes. Naunyn Schmiedebergs Arch Pharmacol 382: 303-310, 2010

65. Meng L, Zhou J, Sasano H, Suzuki T, Zeitoun KM and Bulun SE: Tumor necrosis factor alpha and interleukin 11 secreted by malignant breast epithelial cells inhibit adipocyte differentiation by selectively down-regulating CCAAT/enhancer binding protein alpha and peroxisome proliferator-activated receptor gamma: mechanism of desmoplastic reaction. Cancer Res 61: 2250-2255, 2001.

66. Ruan H, Pownall HJ and Lodish HF: Troglitazone antagonizes tumor necrosis factor-alpha-induced reprogramming of adipocyte gene expression by inhibiting the transcriptional regulatory functions of NF-kappaB. J Biol Chem 278: 28181-28192, 2003.

67. Ritchie SA, Ewart MA, Perry CG, Connell JM and Salt IP: The role of insulin and the adipocytokines in regulation of vascular endothelial function. Clin Sci (Lond) 107: 519-532, 2004.

68. Hotamisligil GS, Shargill NS and Spiegelman BM: Adipose expression of tumor necrosis factor-alpha: direct role in obesity-linked insulin resistance. Science 259: 87-91, 1993.

69. Uysal KT, Wiesbrock SM, Marino MW and Hotamisligil GS Protection from obesity-induced insulin resistance in mice lacking TNF-alpha function. Nature 389: 610-614, 1997.

70. Hotamisligil GS, Peraldi P, Budavari A, Ellis R, White MF and Spiegelman BM: IRS-1-mediated inhibition of insulin receptor tyrosine kinase activity in TNF-alpha- and obesity-induced insulin resistance. Science 271: 665-668, 1996.

71. Shi H, Tzameli I, Bjørbaek C and Flier JS: Suppressor of cytokine signaling 3 is a physiological regulator of adipocyte insulin signaling. J Biol Chem 279: 34733-34740, 2004.

72. Jager J, Grémeaux T, Cormont M, Le Marchand-Brustel Y and Tanti JF: Interleukin-1beta-induced insulin resistance in adipocytes through down-regulation of insulin receptor substrate-1 expression. Endocrinology 148: 241-251, 2007.

73. Griendling KK, Sorescu D and Ushio-Fukai M: NAD(P)H oxidase: role in cardiovascular biology and disease. Circ Res 86: 494-501, 2000

74. Xue X, Piao JH, Nakajima A, et al: Tumor necrosis factor alpha (TNFalpha) induces the unfolded protein response (UPR) in a reactive oxygen species (ROS)-dependent fashion, and the UPR counteracts ROS accumulation by TNFalpha. J Biol Chem 280: 33917-33925, 2005.

75. Li WW, Alexandre S, Cao X and Lee AS: Transactivation of the grp78 promoter by $\mathrm{Ca}^{+}$depletion. A comparative analysis with A23187 and the endoplasmic reticulum $\mathrm{Ca}\left(2^{+}\right)$-ATPase inhibitor thapsigargin. J Biol Chem 268: 12003-12009, 1993.
76. Tsujino M, Hirata Y, Imai T, et al: Induction of nitric oxide synthase gene by interleukin-1 beta in cultured rat cardiocytes. Circulation 90: 375-383, 1994.

77. Cole BK, Kuhn NS, Green-Mitchell SM, etal: 12/15-Lipoxygenase signaling in the endoplasmic reticulum stress response. Am J Physiol Endocrinol Metab 302: E654-E665, 2012.

78. Zhou L and Liu F: Autophagy: roles in obesity-induced ER stress and adiponectin downregulation in adipocytes. Autophagy 6: $1196-1197,2010$

79. Pfaffenbach KT, Gentile CL, Nivala AM, Wang D, Wei Y and Pagliassotti MJ: Linking endoplasmic reticulum stress to cell death in hepatocytes: roles of C/EBP homologous protein and chemical chaperones in palmitate-mediated cell death. Am J Physiol Endocrinol Metab 298: E1027-E1035, 2010.

80. Zhou QG, Zhou M, Hou FF and Peng X: Asymmetrical dimethylarginine triggers lipolysis and inflammatory response via induction of endoplasmic reticulum stress in cultured adipocytes. Am J Physiol Endocrinol Metab 296: E869-E878, 2009.

81. Deng J, Liu S, Zou L, Xu C, Geng B and Xu G: Lipolysis response to endoplasmic reticulum stress in adipose cells. J Biol Chem 287: 6240-6249, 2012.

82. Cnop M, Foufelle F and Velloso LA: Endoplasmic reticulum stress, obesity and diabetes. Trends Mol Med 18: 59-68, 2012

83. Boden G, Lebed B, Schatz M, Homko C and Lemieux S: Effects of acute changes of plasma free fatty acids on intramyocellular fat content and insulin resistance in healthy subjects. Diabetes 50: 1612-1617, 2001.

84. Reaven GM, Hollenbeck C, Jeng CY, Wu MS and Chen YD Measurement of plasma glucose, free fatty acid, lactate, and insulin for $24 \mathrm{~h}$ in patients with NIDDM. Diabetes 37: 1020-1024, 1988.

85. Nguyen MT, Satoh H, Favelyukis S, et al: JNK and tumor necrosis factor-alpha mediate free fatty acid-induced insulin resistance in 3T3-L1 adipocytes. J Biol Chem 280: 35361-35371, 2005.

86. Gao Z, Hwang D, Bataille F, et al: Serine phosphorylation of insulin receptor substrate 1 by inhibitor kappa B kinase complex. J Biol Chem 277: 48115-48121, 2002.

87. Haukeland JW, Dahl TB, Yndestad A, et al: Fetuin A in nonalcoholic fatty liver disease: in vivo and in vitro studies. Eur J Endocrinol 166: 503-510, 2012

88. Arner P: Insulin resistance in type 2 diabetes: role of fatty acids. Diabetes Metab Res Rev 18 (Suppl 2): S5-S9, 2002.

89. Jensen MD: Adipose tissue metabolism - an aspect we should not neglect? Horm Metab Res 39: 722-725, 2007.

90. Gregor MF and Hotamisligil GS: Thematic review series: Adipocyte Biology. Adipocyte stress: the endoplasmic reticulum and metabolic disease. J Lipid Res 48: 1905-1914, 2007.

91. Bollheimer LC, Skelly RH, Chester MW, McGarry JD and Rhodes CJ: Chronic exposure to free fatty acid reduces pancreatic beta cell insulin content by increasing basal insulin secretion that is not compensated for by a corresponding increase in proinsulin biosynthesis translation. J Clin Invest 101: 1094-1101, 1998.

92. Liu YQ, Jetton TL and Leahy JL: beta-Cell adaptation to insulin resistance. Increased pyruvate carboxylase and malate-pyruvate shuttle activity in islets of nondiabetic Zucker fatty rats. J Biol Chem 277: 39163-39168, 2002.

93. Prentki $\mathrm{M}$ and Nolan CJ: Islet beta cell failure in type 2 diabetes. J Clin Invest 116: 1802-1812, 2006

94. McGarry JD and Dobbins RL: Fatty acids, lipotoxicity and insulin secretion. Diabetologia 42: 128-138, 1999.

95. Kharroubi I, Ladrière L, Cardozo AK, Dogusan Z, Cnop M and Eizirik DL: Free fatty acids and cytokines induce pancreatic beta-cell apoptosis by different mechanisms: role of nuclear factor-kappaB and endoplasmic reticulum stress. Endocrinology 145: 5087-5096, 2004.

96. Cunha DA, Hekerman P, Ladrière L, et al: Initiation and execution of lipotoxic ER stress in pancreatic beta-cells. J Cell Sci 121: 2308-2318, 2008

97. Ryden M, Dicker A, van Harmelen V, et al: Mapping of early signaling events in tumor necrosis factor-alpha -mediated lipolysis in human fat cells. J Biol Chem 277: 1085-1091, 2002.

98. Fricke K, Heitland A and Maronde E: Cooperative activation of lipolysis by protein kinase $\mathrm{A}$ and protein kinase $\mathrm{C}$ pathways in 3T3-L1 adipocytes. Endocrinology 145: 4940-4947, 2004.

99. Kawasaki N, Asada R, Saito A, Kanemoto S and Imaizumi K Obesity-induced endoplasmic reticulum stress causes chronic inflammation in adipose tissue. Sci Rep 2: 799, 2012.

100. Malhotra JD and Kaufman RJ: Endoplasmic reticulum stress and oxidative stress: a vicious cycle or a double-edged sword? Antioxid Redox Signal 9: 2277-2293, 2007. 